\title{
Silicon-Integrated Hybrid-Vertical-Cavity Lasers for Life Science Applications
}

\author{
J.S. Gustavsson ${ }^{a \star}$, S. Kumarib,c, E.P. Haglund ${ }^{\mathrm{a}}$, J. Bengtsson ${ }^{\mathrm{a}}$, G. Roelkens ${ }^{\mathrm{b}, \mathrm{c}}$, R.G. Baets ${ }^{\mathrm{b}, \mathrm{c}}$, and A. \\ Larsson $^{\mathrm{a}}$, \\ aPhotonics Laboratory, Department of Microtechnology and Nanoscience, Chalmers University of \\ Technology, SE-41296, Göteborg, Sweden, bPhotonics Research Group, INTEC Department, Ghent \\ University-IMEC, Belgium, ${ }^{\mathrm{C}}$ Center for Nano- and Biophotonics, Ghent University
}

*iohan.gustavsson@chalmers.se

The integration of efficient laser sources on silicon would enable fully integrated silicon photonic circuits with a high degree of functionality and performance complexity for many applications ${ }^{1}$. Different integration concepts have therefore been suggested, where one such technique is the heterogeneous integration of a vertical-cavity laser (VCL), referred to as a hybrid VCL. It is promising as it has potential to offer low drive currents, high modulation bandwidths, and small footprint ${ }^{2-4}$. In-plane emission with waveguide-coupling can be achieved by an intra-cavity waveguide embossed with a weak diffraction grating, as an example ${ }^{5}$. Integration of such short-wavelength laser sources on a silicon-nitride (SiN) waveguide platform on silicon may enable fully integrated silicon photonic circuits for applications not only in short-reach optical interconnects but also in life science and bio-photonics. Most biological species and processes are probed in the visible and near-infrared (400-1000 nm wavelengths), and of particular interest is the therapeutic window in the very-near-infrared (750-930 nm wavelengths) where there is minimal photo-damage to cells and negligible water absorption.

As a first step in realizing short-wavelength hybrid VCLs with in-plane emission coupled to a SiN waveguide, we have developed a technique to produce high performance 850-nm hybrid VCLs with outof-plane emission. It is based on adhesive bonding of epitaxial AlGaAs-material onto a dielectric distributed Bragg reflector (DBR) on silicon ${ }^{6-8}$. We have fabricated devices with surface emission having sub-mA threshold current, $>2 \mathrm{~mW}$ output power, and $25 \mathrm{Gbit} / \mathrm{s}$ modulation speed ${ }^{8}$. We have also shown experimentally that the bonding layer thickness can be used to optimize a certain performance parameter at a given temperature or to minimize the variation of performance over temperature ${ }^{8}$.

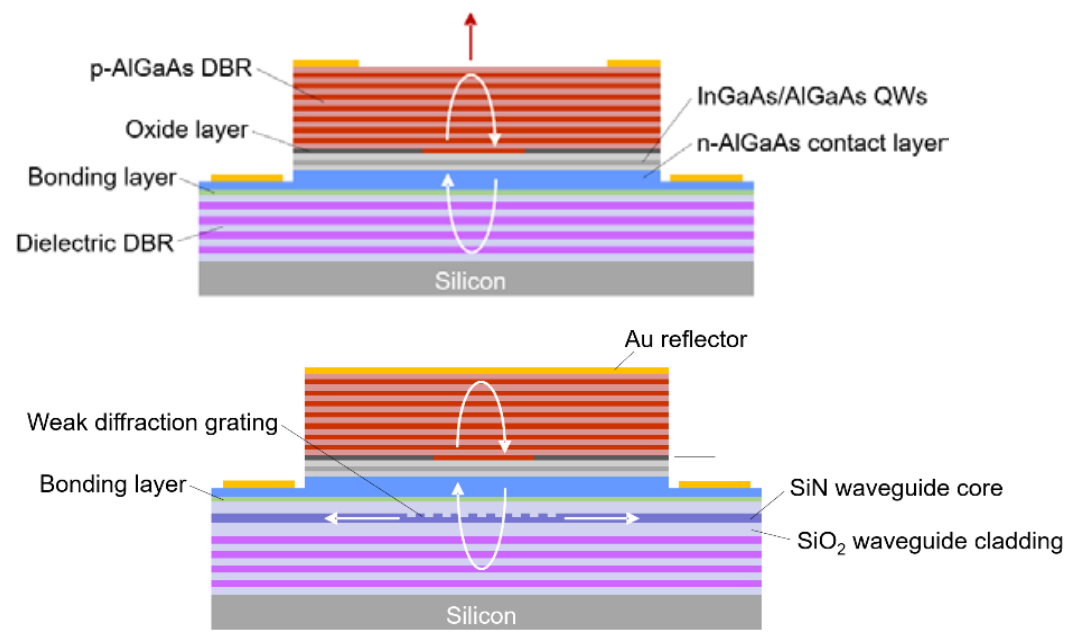

Fig.1 Schematic cross-section of our 850-nm hybrid VCLs: surface-emitting design (top), and in-plane emitting design with SiNwaveguide coupling (bottom).

Our top-emitting hybrid VCL design is shown in Fig.1, where the AlGaAs-material consists (from bottom to top) of an n-doped AIGaAs contact/current spreading layer, an active region with five 4-nm-thick InGaAs/AIGaAs quantum wells (QWs), a 30-nm-thick p-doped Alo.98Ga0.02As layer for the formation of an oxide aperture, and a p-doped 23 pair AIGaAs DBR. The dielectric DBR deposited on Silicon is a 20pair $\mathrm{SiO}_{2} / \mathrm{Ta}_{2} \mathrm{O}_{5} \mathrm{DBR}$. The bonding layer consists of a thin layer of $\mathrm{SiO}_{2}$ (deposited on the dielectric DBR) and an ultra-thin layer of divinylsiloxane-bis-benzocyclobutene (DVS-BCB). The DVS-BCB layer is used as the adhesive bonding agent ${ }^{9}$, and its thickness is kept constant while the thickness of the $\mathrm{SiO}_{2}$ layer is used to control the bonding layer thickness. Fig. 2 shows scanning electron microscopy (SEM) images of a device cross-section, and measured steady-state characteristics for a $10 \mu \mathrm{m}$ oxide aperture device with a $\sim 65-\mathrm{nm}$-thick bonding layer, resulting in an $\sim 853 \mathrm{~nm}$ resonance wavelength at $25^{\circ} \mathrm{C}$. The slope efficiency is $\sim 0.55 \mathrm{~W} / \mathrm{A}$ at $25^{\circ} \mathrm{C}$. 

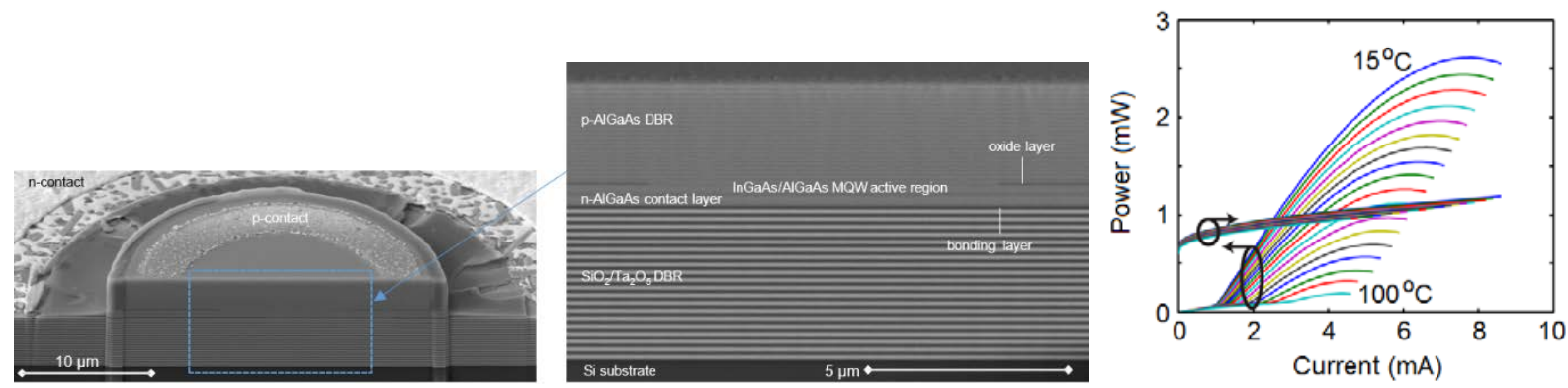

Fig. 2 SEM images of fabricated top-emitting hybrid VCL cross-section after focused ion beam etching (left and middle), and measured steady-state optical output power and voltage drop versus drive current and ambient temperature for a device with a $\sim 65 \mathrm{~nm}$ bonding layer thickness and $10 \mu \mathrm{m}$ oxide aperture (right).

To be able to demonstrate in-plane emission with SiN waveguide coupling from our hybrid 850-nm VCLs, our next step is to add a SiN waveguide structure with embossed grating on top of the dielectric DBR, before adhesively bonding the AIGaAs-material (Fig. 1, bottom). So far, based on numerical simulations, we have designed a device that is predicted to yield a slope efficiency of $\sim 0.3$ W/A at $25^{\circ} \mathrm{C}$ for the light coupled to a single-mode waveguide, while maintaining a sub-mA threshold current for the lasing ${ }^{10}$. The single-mode SiN waveguide core is 300-nm-thick and 500-nm-wide. Since the cavity field is much wider (defined by oxide aperture) than the width of the single-mode waveguide, a tapering of the waveguide width is needed between the grating and the single-mode waveguide. The design work consisted of three parts, where the first part was to investigate (using FIMMWAVE optical mode solver) the required thickness of the top and bottom $\mathrm{SiO}_{2}$ waveguide cladding layers to prevent guided light in the waveguide to leak away into the high index AIGaAs-material or dielectric DBR and Si substrate. The second part was to compute (using Lumerical 2D-FDTD analysis) the fraction of the light incident on the grating and structure below that is coupled into the SiN waveguide. With this information, a final third part (using 1D TMM calculations) was performed to calculate the threshold gain and estimate the slope efficiency. As can be seen in Fig. 3, a slope efficiency $>0.3$ W/A can be achieved for a threshold gain $<1000 \mathrm{~cm}^{-1}$, which is required for sub-mA threshold currents.
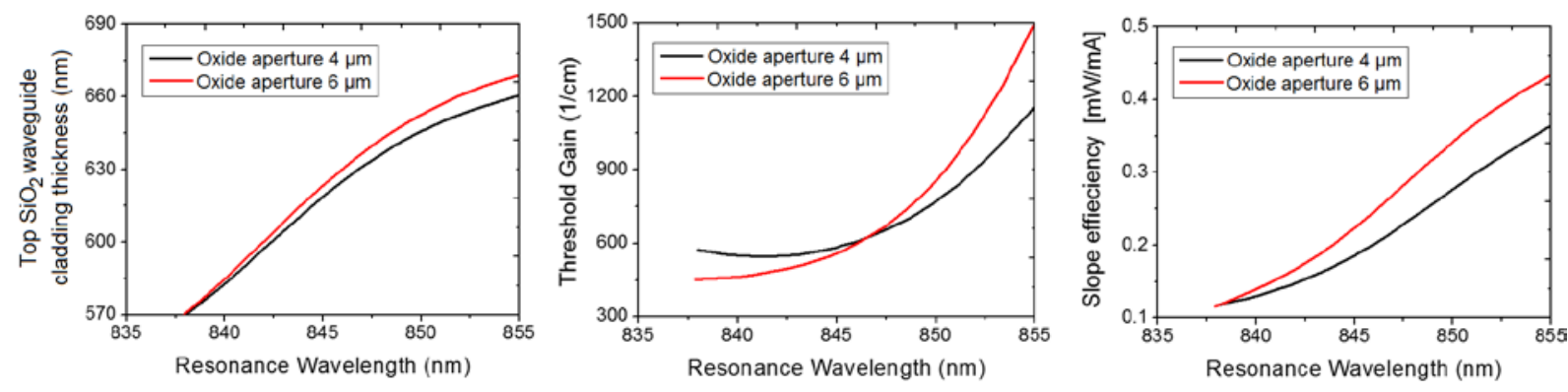

Fig.3 Calculated resonance wavelength, threshold gain, and slope efficiency versus top $\mathrm{SiO}_{2}$ waveguide cladding thickness for our 850-nm-wavelength hybrid VCL design with in-plane coupling to a single-mode SiN-waveguide. The bottom $\mathrm{SiO}_{2}$ waveguide cladding thickness is $900 \mathrm{~nm}$, and the grating period, duty cycle, and etch depth are $525 \mathrm{~nm}, 50 \%$, and 30 nm, respectively.

This work was supported by the European Union's Horizon 2020 research and innovation program under grant agreement no. 688519 (PIX4life), the Swedish Foundation for Strategic Research (SSF), and the European FP7-ERC-InSpectra Advanced Grant.

\section{References:}

1. Z. Zhou et al., Light Sci. Appl., vol. 4, no. 11, p. e358 (2015)

2. Tsunemi et al., Opt. Express, vol. 21, no. 23, p. 28685 (2013)

3. J. Ferrara et al., Opt. Express, vol. 23, no. 3, p. 2512 (2015)

4. G.C. Park et al., Laser Photon. Rev., vol. 9, no. 3, p. L11 (2015)

5. D. A. Louderback et al., Electron. Lett., vol. 40, no. 17, p. 1064 (2004)

6. E.P. Haglund et al., Opt. Express, vol. 23, no. 26, p. 33634 (2015)

7. E.P. Haglund et al., IEEE Photon. Technol. Lett., vol. 28, no. 8, p. 856 (2016)

8. E.P. Haglund et al., IEEE J. Sel. Top. Quantum Electron., vol. 23, no. 6, p. 1700109 (2017)

9. S. Keyvaninia et al., Opt. Mater. Express, vol. 3, no. 1, p. 35 (2013)

10.S. Kumari et al., Submitted to Optics Express (2017) 